RESEARCH PAPER RP1223

Part of Journal of Research of the National Bureau of Standards, Volume 23, July 1939

\title{
DETECTION OF RADIOACTIVE CONTAMINATION, USING GEIGER-MÜLLER COUNTERS
}

\author{
By Leon F. Curtiss
}

\section{ABSTRACT}

Situations frequently arise in which it is desirable to determine the extent of accidental contamination by radioactive materials. Such contamination occurs in research laboratories where radioactive studies are in progress and also in commercial plants where radioactive materials are handled. An important example of the latter is the dial-painting plants, where self-luminous radium paint is applied.

This article describes a portable Geiger-Müller counter operated entirely from the alternating-current mains, which permits a rapid and accurate determination of such contamination wherever it exceeds the equivalent of about one-half microgram of radium per square meter. The device is also sufficiently sensitive to determine the presence of radium in amounts of micrograms in a living person and therefore may be used for routine test of workers, their garments, and objects habitually handled by them. The instrument is very rugged, readily portable, and silent in operation. Requiring no batteries of any kind, it is always ready for use. Commercial radio parts are used well within their ratings throughout, with the exception of the tube counter itself, so that there is little danger of failures, and the parts are easily replaced if they should fail. The indicator is a milliammeter, which is arranged to have a very steady deflection under constant conditions and is yet sufficiently sensitive to measure 1 microgram of radium at a distance of 1 meter. The device may therefore be used for intercomparison by gamma radiation of samples of radium of low activity. It has been tested under practical conditions and has been found well suited for surveys of contaminated locations. It also is a very sensitive detector for finding lost radium preparations and for testing radium ores for commercial value.

\section{CONTENTS}

I. Introduction

II. Power-supply unit. 138

III. Amplifier unit.

IV. Counter tube

V. Assembly and use of counter equipment

VI. Surveying for contamination

\section{INTRODUCTION}

Recently the writer has been called upon to determine the radioactive contamination present in several of the more important radium dial-painting plants, and also to design and construct 10 instruments for the Navy Department that would be suitable for similar surveys in their dial-painting plants. A sensitive portable Geiger-Müller counter seemed most suitable for this work. Equipment for such work should be capable of calibration and of maintaining its calibration over 
periods of months or years. For most effective results, it should be entirely silent in operation. Furthermore, it should be rugged in construction. In the absence of any existing apparatus which entirely fulfilled these conditions, the writer undertook to design a counter unit which could be operated entirely from the alternating-current supply and would satisfy the above conditions. A description of the instrument is given below, with examples of its use in various parts of the country over a period of months, during which it has maintained its original calibration with surprising fidelity. Commercial radio parts, used well within their ratings, are employed throughout, except for the tube counter itself. Thus repairs, should they be required, may be quickly and simply made.

\section{POWER-SUPPLY UNIT}

For ease in handling, it was decided to build the equipment in two units, (1) a power supply to furnish the high voltage for the counter and the various plate and grid bias voltages for the amplifier, and (2)

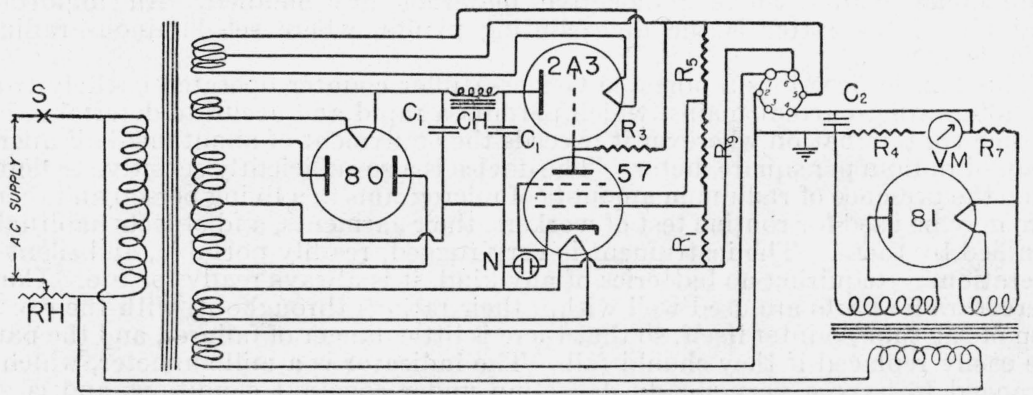

Figure 1.-Wiring diagram of the power-supply unit.

$N$, 1/4-watt neon bulb; $C H$, 12-henry choke; $R_{1}, 11,000$ ohms; $R_{3}, 0.5$ megohm; $R_{4}, 1$ megohm; $R_{5}, 10,000$ ohms; $R_{7}, 100,000 \mathrm{ohms} ; R_{9}, 27,000 \mathrm{ohms} ; C_{1}, 8$ microfarads; and $C_{2}, 4$ microfarads.

an amplifier unit which contains the tube counter and an amplifier to make its indications visible by means of a milliammeter.

The wiring diagram of the power supply is shown in figure 1 . The amplifier voltages are supplied by the self-regulating voltage supply, which contains the type $80,2 \mathrm{~A} 3$, and 57 vacuum tubes with a neon lamp, $N$, to provide a constant bias for the 57 tube. This type of voltage supply has been described by Grammer. ${ }^{1}$ It is so arranged that the input-line voltage can fluctuate from about 85 to 135 volts without changing the plate voltage by more than 1 volt. The counter voltage is supplied by the simple rectifier circuit, using a type 81 rectifier and an $8-\mu f$ filter condenser. The voltage is read by a milliammeter with a megohm resistor, $R_{4}$, in series. The operating voltage for the counter is 600 volts and is controlled by the rheostat, $R H$. The power supply is built into a metal cabinet. Figure 2 shows this cabinet with its sides removed to show the arrangement of parts.

\section{AMPLIFIER UNIT}

The wiring diagram of the amplifier is shown in figure 3 . The counter, $G-M$, is connected directly to the first 57 tube, which is arranged to form a Neher and Harper ${ }^{2}$ circuit. This circuit is used

1 G. Grammer, QST 21, 14 (August 1937).

2 Phys. Rev. 47, 940 (1936). 


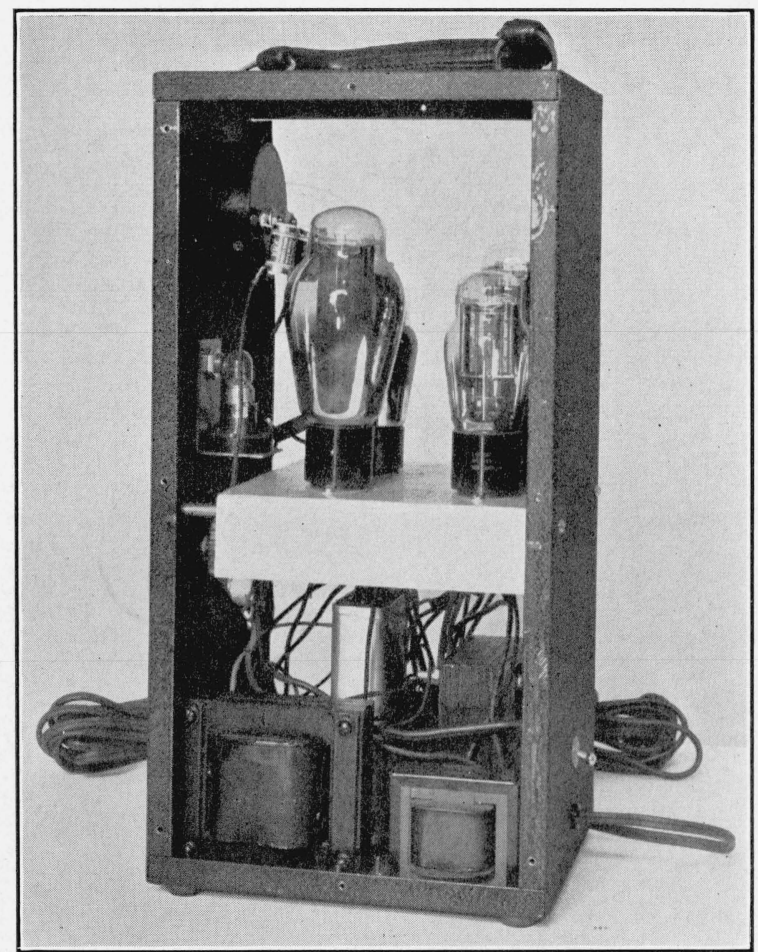

FIGURE 2.-Power-supply unit, with sides of cabinet removed. 


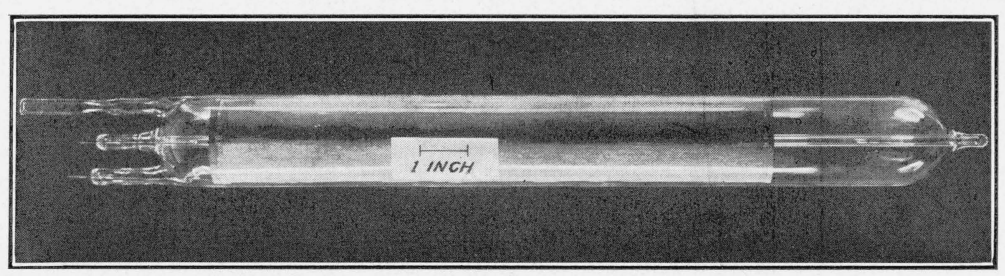

Figure 4.-Tube counter. 
because of its high speed, being a much faster circuit than the Neher and Pickering circuit. ${ }^{3}$ For a discussion of reasons for the relative speeds of these two circuits, see Kozodaev and Latyschev. ${ }^{4}$ Since the pulses from a Neher and Harper circuit are not of uniform height, it is necessary to level them if an integrating circuit is to be used. This is accomplished by the second 57 tube. The levelled pulses are rectified by the 27 tube and charge the condenser $C_{4}$, connected to the plate of the 27 tube through a resistor, $R_{7}$, and also to the grid of the 56 tube. This tube is connected to serve as a vacuum-tube voltmeter to read the voltage on the condenser, $C_{4}$. The grid of the 56 tube is also connected to the cathode through resistors, as shown, so that for a given rate of charge the condenser acquires a steady negative voltage. This is indicated by a steady deflection of the meter, $M$, in the plate circuit of the 56 tube. To avoid excessive fluctuation of this potential

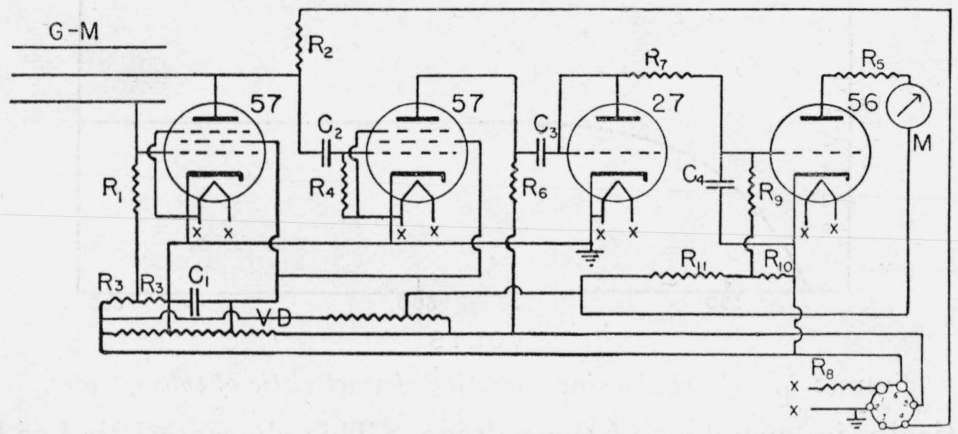

Figure 3.-Wiring diagram of the amplifier used with the tube counter.

$G M$, tube counter; $V D$ voltage divider; $R_{1}, R_{7}, 20$ megohms; $R_{2}, R_{4}, R_{9}, 5$ megohms; $R_{3}, 54000$ ohms; $R_{5}$, 10,000 ohms; $R_{6}, 0.5$ megohm; $R_{8}, 1 \mathrm{ohm} ; R_{10}, 2,000 \mathrm{ohms} ; R_{11}, 0.25$ ohm; $\mathrm{C}_{1}, 8$ microfarads; $C_{2}, 0.0001$ microfarad and $C_{3}, 0.0002$ microfarad.

as a result of statistical fluctuations in the counting rate of the tube counter, a relatively large counter is used, so that its rate, even in the absence of a radioactive sample, is fast enough to give a sensibly steady reading. This stability is of course partly due to the large capacity, $C_{4}$, and relatively low resistance across it, $R_{9}$ plus $R_{10}$. For the complete range of the indicating meter, the actual counting rate of the tube counter changes from about 600 to 10,000 per minute.

\section{COUNTER TUBE}

To secure the rapid counting rate required, the counter tube shown in figure 4 has been adopted. It is approximately $5 \mathrm{~cm}$ in diameter and $30 \mathrm{~cm}$ long, using a 100 by 100 mesh copper-gauze cylindrical electrode, as recommended by Evans. ${ }^{5}$ The general construction, as can be seen from the photograph, is that described by Cosyns and de Bruyn, ${ }^{6}$ using glass sleeves as guard rings on the wire electrode. Since it is desirable to operate the counter at a low voltage to reduce insulation problems and decrease the excess potential on the Neher and Harper circuit, these counters are filled with a mixture of argon and

\footnotetext{
3 Phys. Rev. 53, 316 (1938).

4 M. Kozodaev and G. C. Latyschev, Compt. rend. (Doklady) acad. Sci. USSR 20, 21, (1938).

5 Rev. Sci. Inst. 7, 444 (1936)

${ }^{6}$ A cad. roy. Belg. Sei. Bul. 20, 371 (1934).
} 
hydrogen at a pressure of approximately $6 \mathrm{~cm}$ of $\mathrm{Hg}$. They require 600 volts to operate near the middle of their plateau. To secure a broad and sensibly level plateau, it has been found necessary to sputter the counters. This treatment is a modification of the method described by Diffenbach, Lifschutz, and Slawsky. ${ }^{7}$ The electrodes are connected to a transformer adjusted to produce a steady glow between the electrodes in hydrogen at a pressure of a few millimeters. The hydrogen is changed frequently and the sputtering permitted to continue for from 12 to 24 hours. Care must be taken to prevent any actual sputtering of the metal which may be deposited on the interior surfaces of the glass and ruin the insulation. Counters prepared in this way have a characteristic voltage curve, as shown in the graph in figure 5. Within a range of about 200 volts their rate is

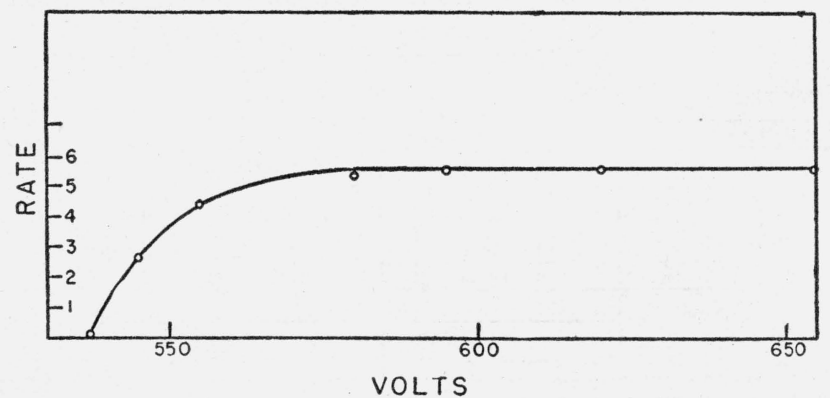

FIGURE 5.-Curve showing operating characteristic of tube counter.

practically independent of the voltage. This characteristic has been determined in integrating circuits of the type described above and also in scaling circuits where actual counts are scaled numerically. The curves obtained are nearly identical in shape, indicating that the integrating circuit delivers uniform pulses to the integrating condenser. This has also been checked with a cathode-ray oscillograph.

In actual use it is necessary to protect the tube counter from breakage, screen it from stray light, and provide an electrostatic shield. These are accomplished by mounting it inside a close-fitting Duralumin tube with walls about $1 / 16$ inch thick. Rubber supports are provided to act as shock absorbers. This metal tube acts as a filter to screen out most of the beta radiation. Figure 6 shows the interior parts of the amplifier and the method of supporting the tube counter within its tubular shield. The case is a rugged Duralumin box cast in one piece. Since surfaces, as tops of tables and floors, are most common objects for investigating contamination, the tube counter is mounted below the case, where it is well protected but also directly accessible from three sides. The asymmetrical position of the metal tube permits the mounting of the active volume of the counter in a symmetrical position with reference to the case.

\section{ASSEMBLY AND USE OF COUNTER EQUIPMENT}

Each of the two cabinets is provided with a socket into which a five-conductor cable, with suitable fittings, may be plugged. This cable is about 20 feet long, so that a considerable area may be surveyed without moving the power-supply cabinet. This cabinet is

\footnotetext{
7 Phys. Rev. 52, 1931 (1937).
} 


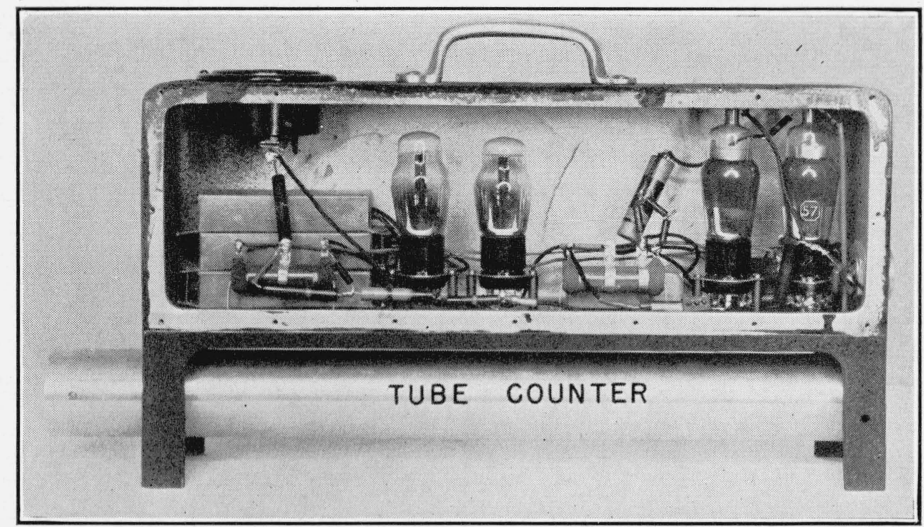

FIGURE 6.-Amplifier unit with side removed and with tube counter in position.

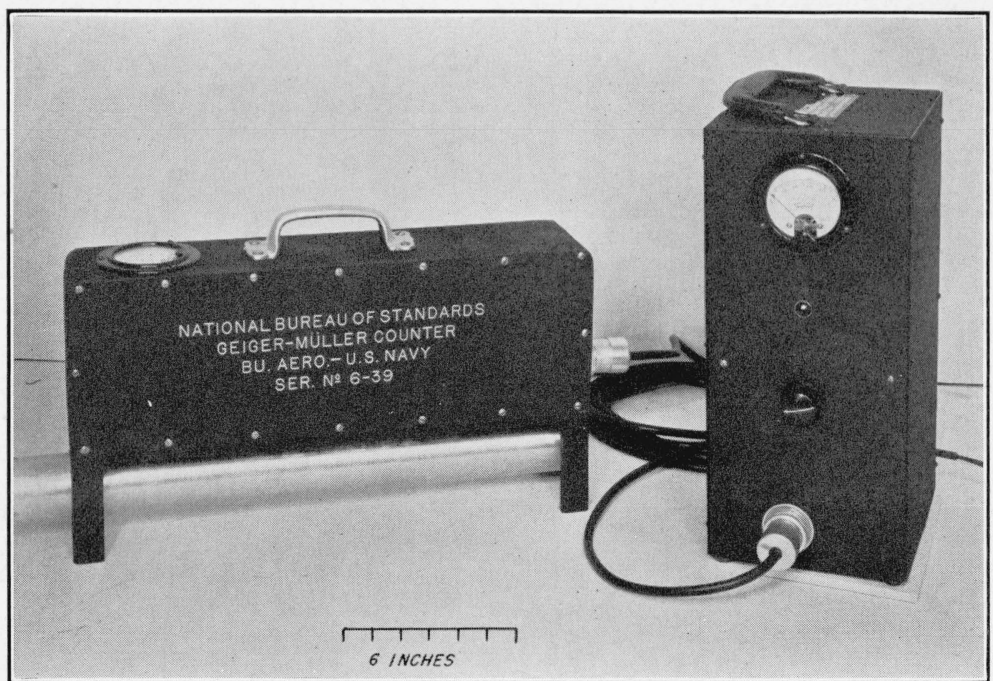

Figure 7.-Complete apparatus assembly ready for use. 
somewhat heavy, inasmuch as all of the transformers are mounted in it.

Figure 7 is a photograph of the complete assembly, with the power supply on the right. The only control to adjust in the use of the instrument is the rheostat knob shown below the voltmeter, which is used to set the voltmeter to a reading of $600 \mathrm{volts}$. This also insures that tube filaments and heaters and bias voltages are the same as at the time of adjustment and calibration.

It has been found that this equipment can be calibrated and will hold its calibration to within better than 10 percent over a period of several months. A typical calibration curve is shown in figure 8 ,

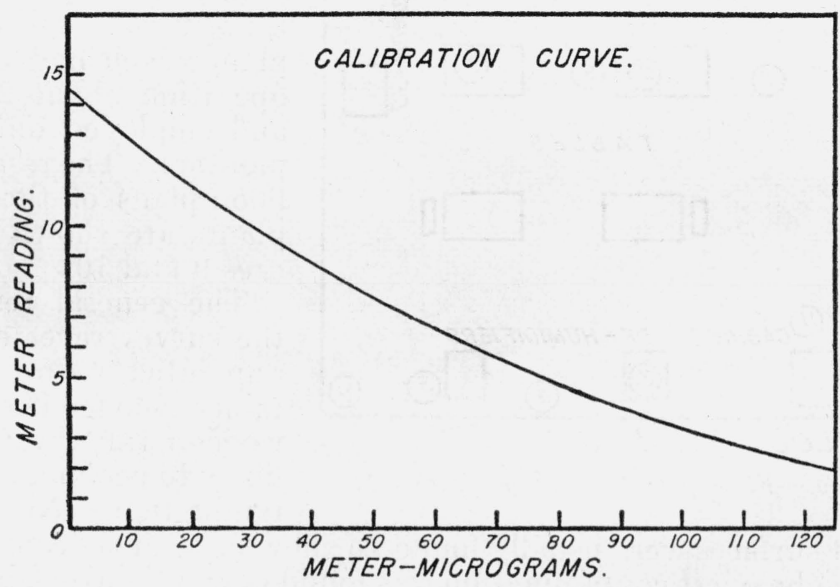

FIGURE_8.-Calibration curve.

where the reading of the meter is plotted against the intensity of gamma-radiation expressed in "meter-micrograms." Thus the numbers along the $X$-axis represent the number of micrograms of radium, at a distance of 1 meter, required to produce the corresponding reading on the milliammeter. The meter used has 75 divisions and can be read to the nearest division with certainty. A deflection of four divisions corresponds roughly to 2.5 meter-micrograms, so that 0.15 microgram will produce this deflection when placed at a distance of $25 \mathrm{~cm}$.

It is possible to adjust this equipment to a greater sensitivity when desirable. In practical use, however, it has been found that an increased sensitivity makes it impossible to obtain readings in heavily contaminated areas. One convenient way to control the sensitivity is to change the size of the coupling condenser, $C_{2}$, shown in the wiring diagram, figure 3 . In this way intensities up to 500 metermicrograms can be measured. If a multirange instrument is desired this may be accomplished by inserting a switch which will change the coupling capacity by cutting various condensers in or out, so that the coupling is changed by fixed steps.

\section{SURVEYING FOR CONTAMINATION}

The instrument described above has been used in several of the larger dial-painting plants. Measurements were made after all radioactive working materials had been removed. The general pro- 
cedure in making these measurements was to take observations at about 10 to 15 different locations uniformly distributed over the plant and then explore the neighborhood of those showing the highest readings in order to locate definitely the areas or objects with heavy

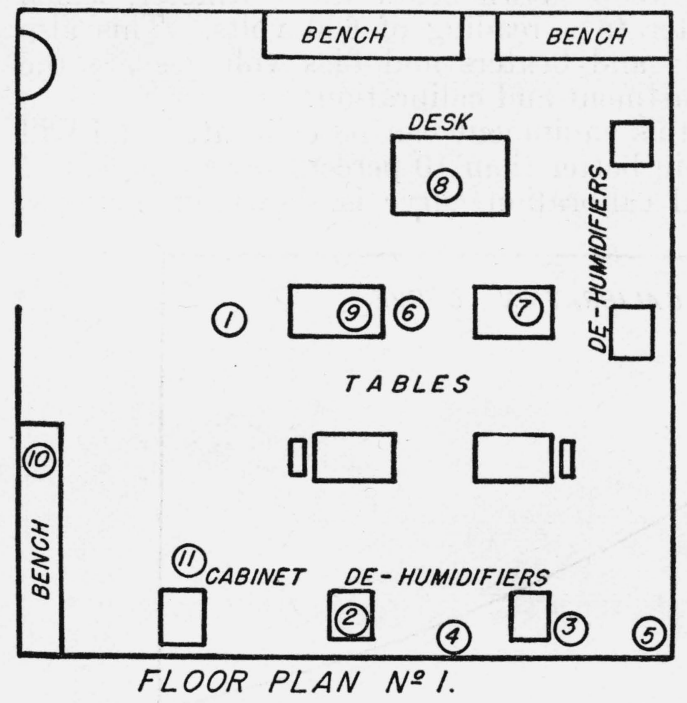

Figure 9.-Floor plan to accompany table 1. contamination. Typical results are shown in tables 1 and 2. The data in table 1 were obtained in a plant which had been in operation for about 5 years and employed about 25 painters. The data in table 2 were taken in a plant which had been in operation about 2 years and employed only four painters. The respective floor plans of these two plants are shown in figures 9 and 10.

The general result of the survey revealed that unpainted wooden-top tables, wooden floors, and wooden trays were most likely to accumulate contamination. Nonporous and metal surfaces were usually found to show only slight contamination. Also heaviest contamination was found near weighing tables and hoods in those plants where the dry luminous powder was weighed out and placed in small containers. This may be attributable to the use of wooden-top tables for this work. Wooden floors also showed much higher contamination than painted concrete floors. Where extreme care was used some wooden floors were cleaner than concrete floors where less care had apparently been exercised.

TABLE 1.-Meter readings in plant 1

\begin{tabular}{|c|c|c|c|}
\hline Location number ${ }^{1}$ & Location & Reading & $\begin{array}{l}\text { Meter- } \\
\text { micro- } \\
\text { grams }\end{array}$ \\
\hline & Center of workroom floor.-. & 1. 6 & 250 \\
\hline & On dehumidifier No. 1 . & 2.7 & 200 \\
\hline & $\begin{array}{l}\text { On dehumidifier after removal of wastebasket containing } \\
\text { wiping cloths. }\end{array}$ & 4.3 & 145 \\
\hline 3 & On dehumidifier No. 2 & 5.1 & 120 \\
\hline & On floor near west wall.......... & 6.9 & \\
\hline 5 & On floor in southwest corner & 8.0 & 37 \\
\hline $6+\ldots . .19$ & On floor near smal! bench. & 4.1 & 150 \\
\hline & 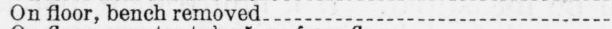 & 6.2 & 90 \\
\hline $6 \ldots$ & On floor, counter tube $5 \mathrm{~cm}$ from floor.. & 5.0 & 120 \\
\hline 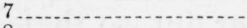 & Top of work table No. 1..................... & 1.8 & 240 \\
\hline 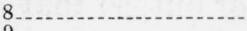 & Top of foreman's desk.................. & 1.7 & 245 \\
\hline 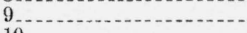 & On work table No. 2 & 1.3 & 265 \\
\hline 10 & 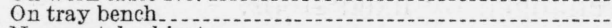 & 2.3 & 217 \\
\hline 11 . & 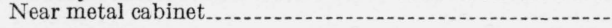 & 0.4 & 325 \\
\hline
\end{tabular}

${ }^{1}$ See figure 9. 
TABLE 2.-Meter readings in plant 2

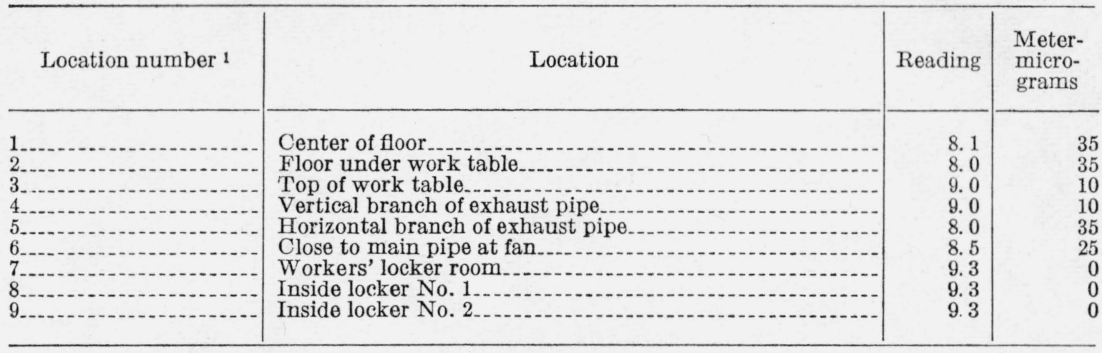

1 See figure 10.

With this equipment it was found that a plant could be completely surveyed in about 2 hours. Approximately 5 minutes is required to obtain a reading at any one point. This is a much shorter time than is usually required with tube counters and is a result of the use of a large counter with a high-speed counting circuit.

Incidental to the tests made with this equipment, several small amounts of radium were discovered which had been misplaced by the owners. One milligram of radium can easily be detected at a distance of about 20 feet, even through intervening walls and other absorbing material. Wherever alternating current can be made available this equipment makes a very satisfactory de-

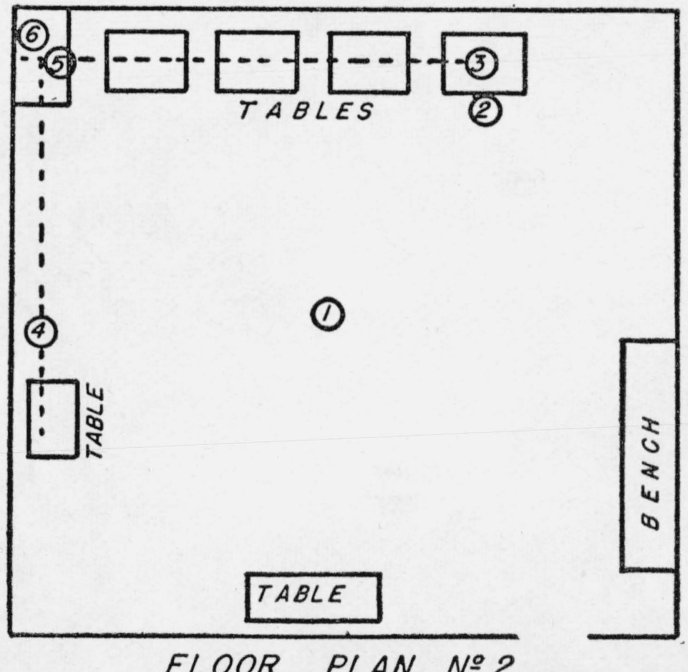

FLOOR PLAN No2.

Figure 10.-Floor plan to accompany table 2. tector for lost radium preparations. It is also sufficiently sensitive to test radium ores which contain sufficient radium to be of commercial value. In situations where alternating current is not available, a portable counter unit may be used operated entirely from dry batteries. Such a unit has been described by the author. ${ }^{8}$

Fifteen instruments have been made in this laboratory, and it was found necessary to make only minor adjustments to bring them all to about the same sensitivity. Calibration curves were then prepared which differed only slightly from each other. With microgram radium standards, such as are now being furnished by the National Bureau of Standards, the instruments may be used for more precise measurements by direct comparison of the unknown with a standard of suitable strength.

Washington, April 14, 1939.

${ }^{8}$ L. F. Curtiss, J. Research NBS 21, 779 (1938) RP1154. 